\title{
DESENVOLVIMENTO DE FERRAMENTA PARA CONTROLE E LANÇAMENTO AUTOMÁTICO DE RESULTADOS ANÁLíTICOS NO LABORATÓRIO DA MINERAÇÃO USIMINAS S.A.*
}

Marco Aurélio de Oliveira Lima' Roberta Fernanda Machado ${ }^{2}$

\section{Resumo}

Este trabalho apresenta uma ferramenta desenvolvida internamente na Mineração Usiminas S.A. em Itatiaiuçu (MG), para o laboratório de análises químicas e físicas de minério de ferro, com os objetivos principais de consolidar os resultados, permitir a gestão das análises em um só local, promover maior rastreabilidade, aumentar a agilidade pelo lançamento automático no sistema SAP ${ }^{\circledR}$ e melhorar a confiabilidade dos resultados obtidos pelo laboratório através da realização de verificações automáticas nos resultados, utilizando-se de comparação com seus históricos e sinalização de resultados incoerentes antes da liberação final.

Palavras-chave:Laboratório; Automatização; Confiabilidade; Reanálise.

\section{DEVELOPMENT OF A TOOL FOR CONTROL AND AUTOMATIC LAUNCH OF ANALYTICAL RESULTS AT MINERAÇÃO USIMINAS S.A. LABORATORY}

\begin{abstract}
This paper presents a tool developed internally at Mineração Usiminas S.A. in Itatiaiuçu (MG), for the chemical and physical analysis of iron ore laboratory, with the main objectives of consolidating the results, making possible the analyzes management in only one place, promoting greater traceability, increasing agility by automatic launching in the SAP® system, and improving the reliability of the results obtained through performing automatic checks on the results, using comparisons with their historical and indicating incoherent results before their final release.
\end{abstract}

Keywords: Laboratory; Automation; Reliability; Reanalysis.

1 Bacharel em Química com atribuições tecnológicas pela Universidade Federal de Uberlândia, Técnico de Laboratório III na Mineração Usiminas. Itaúna, MG. Brasil.

2 Bacharel em Engenharia de Produção pela Universidade Federal de Minas Gerais, Analista de Qualidade na Mineração Usiminas. Itaúna, MG. Brasil 


\section{INTRODUÇÃO}

\subsection{Contextualização}

\subsubsection{O Laboratório de Análises Químicas e Físicas}

O laboratório para o qual foi desenvolvida a ferramenta alvo deste estudo pertence à Mineração Usiminas (MUSA), produtora de minério de ferro localizada na Serra Azul, município de Itatiaiuçu, em Minas Gerais. O laboratório conta atualmente com 22 colaboradores, divididos em três turnos de trabalho, e os principais ensaios realizados são granulometria, umidade, teores de FeT, $\mathrm{SiO}_{2}, \mathrm{Al}_{2} \mathrm{O}_{3}, \mathrm{PPC}$ (Perda Por Calcinação), $\mathrm{P}, \mathrm{Mn}, \mathrm{MgO}, \mathrm{CaO}$, $\mathrm{TiO}_{2}$ e $\mathrm{Fe}_{3} \mathrm{O}_{4}$. As amostras são oriundas de frente de lavra, processos do beneficiamento (alimentação, concentrados e rejeitos), produtos (sinter feed, pellet feed, granulado fino, granulado grosso) e despacho para os clientes. As informações geradas a partir das análises realizadas no laboratório são utilizadas para definições de áreas de lavra, ajustes nos processos produtivos, alterações na blendagem de minérios destinados à venda e cálculo de bônus/penalidades aplicadas em função da qualidade do produto entregue ao cliente.

A demanda do laboratório é de aproximadamente 3.200 amostras/mês com um tempo médio de lançamento manual no sistema SAP ${ }^{\circledR}$ de três minutos por amostra. Antes do desenvolvimento dessa ferramenta, os dados das análises do laboratório eram consolidados manualmente no Microsoft Excel ${ }^{\circledR}$, onde somente a estequiometria da amostra era verificada. Após análise e verificação, a planilha com os dados era impressa, e uma etiqueta padrão para identificação das amostras colada na mesma. Para divulgação dos resultados, os teores eram digitados individualmente pelo analista no sistema SAP ${ }^{\circledR}$.

\subsubsection{Importância da Informação}

Siqueira (2005) afirma que "a informação e o conhecimento tornaramse os principais recursos econômicos para a competitividade das empresas". Com isto, a informação tornou-se um fator fundamental para condução dos negócios e obtenção de competitividade no mercado, sendo fator determinante para orientar as tomadas de decisões (JANNUZZI, FALSARELLA e SUGAHARA, 2014).

É necessário que estas informações sejam disponíveis e confiáveis para que tenham impacto positivo no ambiente empresarial. O tratamento da informação torna-se necessário, e quando utilizado como aporte na tomada de decisões, agrega valor ao negócio, pois possibilita à empresa avaliar oportunidades, ameaças, tendências e problemas (SIQUEIRA, 2005).

\subsubsection{Sistemas de Informação em Laboratórios}

Segundo Santos e Santos (2009), um sistema de informação tem como função facilitar o manuseio e promover rastreabilidade, segurança e confiabilidade, além de reduzir o tempo necessário para a interpretação e disponibilização das informações. 
Para as empresas continuarem crescendo e conseguirem manter agilidade na resolução de problemas e eficácia na tomada de decisão, devem utilizar de sistemas de informação, que podem contribuir para melhor competitividade, redução de custos, agilidade, flexibilidade e velocidade (DIAS e PINTO, 2011).

Inseridos em um ambiente competitivo que almeja maior produtividade, maximização da confiabilidade e minimização de erros, laboratórios têm buscado maior automatização e informatização de seus processos (SANTOS e SANTOS, 2009). É possível encontrar softwares de gestão de informações laboratoriais no mercado. Porém, em função de seu elevado custo, freqüentemente não se apresentam como soluções viáveis para pequenas e médias empresas de mineração, que geralmente optam por desenvolver internamente ferramentas de automatização e informatização, buscando atingir maior produtividade, confiabilidade e velocidade em suas atividades.

Santos e Santos (2009), apresentam como benefícios qualitativos advindos da implantação deste tipo de sistema de informação, "a liberação de um técnico mais especializado das atividades rotineiras para outras atividades que requeiram maior competência e habilidade técnica, padronização dos documentos expedidos pelo laboratório e melhoria do clima organizacional, à medida que as atividades tornam-se menos monótonas e cansativas".

\subsection{Objetivos}

Os objetivos principais ao desenvolver e implantar uma ferramenta para controle e lançamento automático de resultados do laboratório foram consolidar os dados e a gestão das análises em um só local, aumentar a confiabilidade e tornar mais ágil a divulgação das análises realizadas. Para tal, foram trabalhados os seguintes pontos:

a) Minimizar a necessidade de digitação de resultados no sistema SAP $^{\circledR}$;

b) Realizar verificações automáticas nos resultados em relação à estequiometria;

c) Indicar automaticamente os resultados incoerentes das amostras de produção em relação a um histórico;

d) Gerar um arquivo físico padronizado dos resultados e facilitar a rastreabilidade das análises.

\section{MATERIAIS E MÉTODOS}

Para a criação desta ferramenta foram utilizados exclusivamente recursos disponíveis internamente: Os softwares Microsoft Excel ${ }^{\circledR}, \operatorname{SAP}^{\circledR} \mathrm{e}$ SuperQ ${ }^{\circledR}$, além de efetivo próprio.

Esta ferramenta consiste em uma planilha do Microsoft Excel ${ }^{\circledR}$ que pode ser dividida em quatro partes:

A primeira possui campos de identificação da amostra, que deverão ser informados manualmente pelo analista; 
A segunda consiste na alimentação dos resultados, e recebe os dados exportados do software SuperQ ${ }^{\circledR}$, fornecido pelo fabricante do equipamento de Fluorescência de Raios- $X$, dados de análise de ferro por via úmida (massa inicial, volume final e fator de correção), teor de magnetita advindos do equipamento Satmagan ${ }^{\circledR}$ e massas da análise de PPC;

A terceira parte faz uma verificação de fechamento estequiométrico e, para amostras de produtos, realiza a comparação da análise química, teor por teor, com um histórico dos últimos seis meses; Em caso de divergência é sinalizada a necessidade de verificação da coerência do resultado.

A quarta consiste em um processo de exportar os dados obtidos para - SAP ${ }^{\circledR}$ e também gerar um boletim impresso com os resultados das análises para verificações internas e também para fins de auditoria.

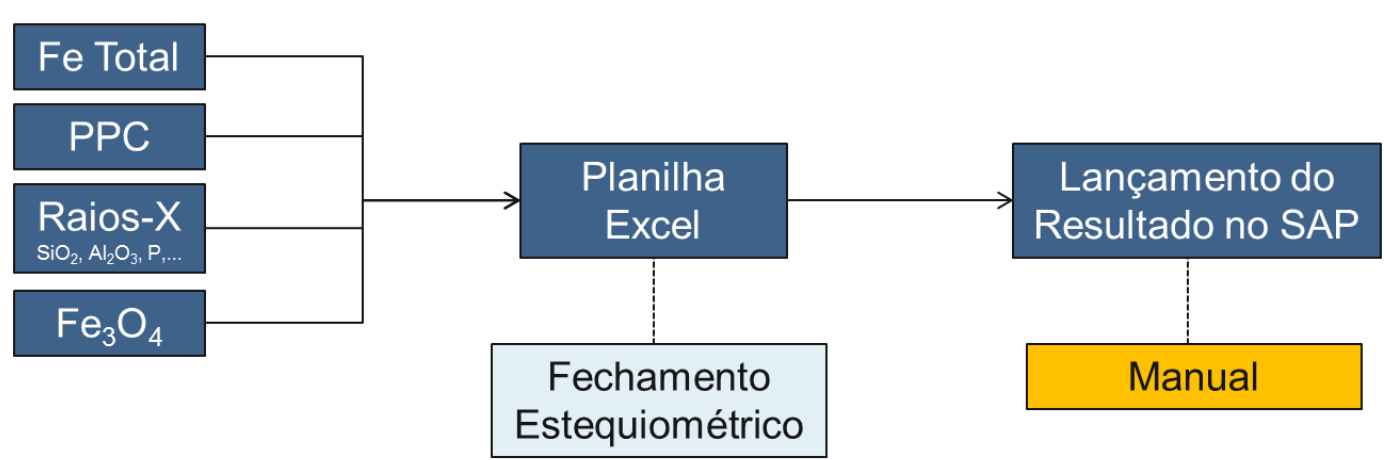

Figura 1 - Fluxo de análise antes da implantação da ferramenta

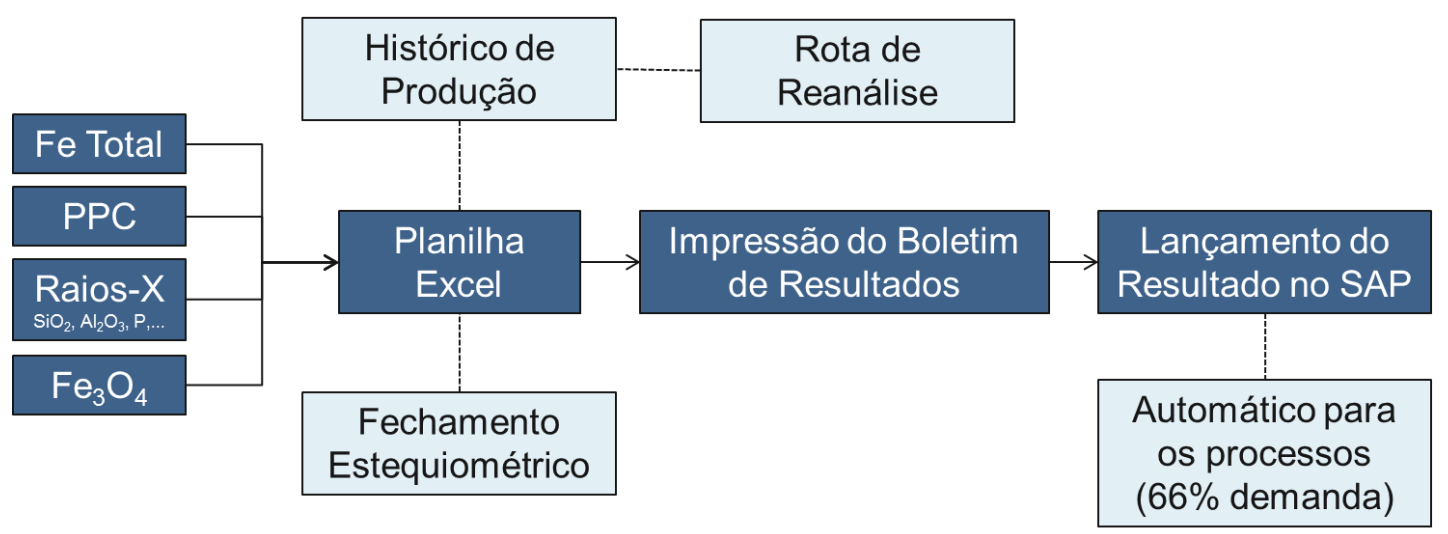

Figura 2 - Fluxo de análise após a implantação da ferramenta

\subsection{Lançamento automático das análises de amostras de processo no sistema SAP ${ }^{\circledR}$}

O lançamento automático dos resultados de análise das amostras de processo no sistema SAP ${ }^{\circledR}$ foi desenvolvido com o objetivo de minimizar a digitação direta pelo analista, reduzindo assim a possibilidade de erros, 0 tempo despendido nesta atividade repetitiva e trazendo mais agilidade ao processo de disponibilização de dados. 
O lançamento ocorre após a execução da validação automática dos dados na planilha Excel ${ }^{\circledR}$, e da seleção por parte do laboratorista de quais amostras serão automaticamente enviadas ao SAP ${ }^{\circledR}$. As informações referentes às amostras selecionadas são exportadas através da geração de um arquivo do tipo CSV (Comma-Separated Values), um formato de armazenamento que as agrupa; $O$ envio de dados para 0 SAP ${ }^{\circledR}$ ocorre através da solicitação do analista, através de uma transação específica nesse sistema.

O esquema abaixo ilustra o processo de identificação, análise, consolidação, verificação, lançamento e disponibilização dos dados para as amostras de processo. As etapas em laranja permanecem manuais, enquanto as caixas em verde representam os processos automáticos.

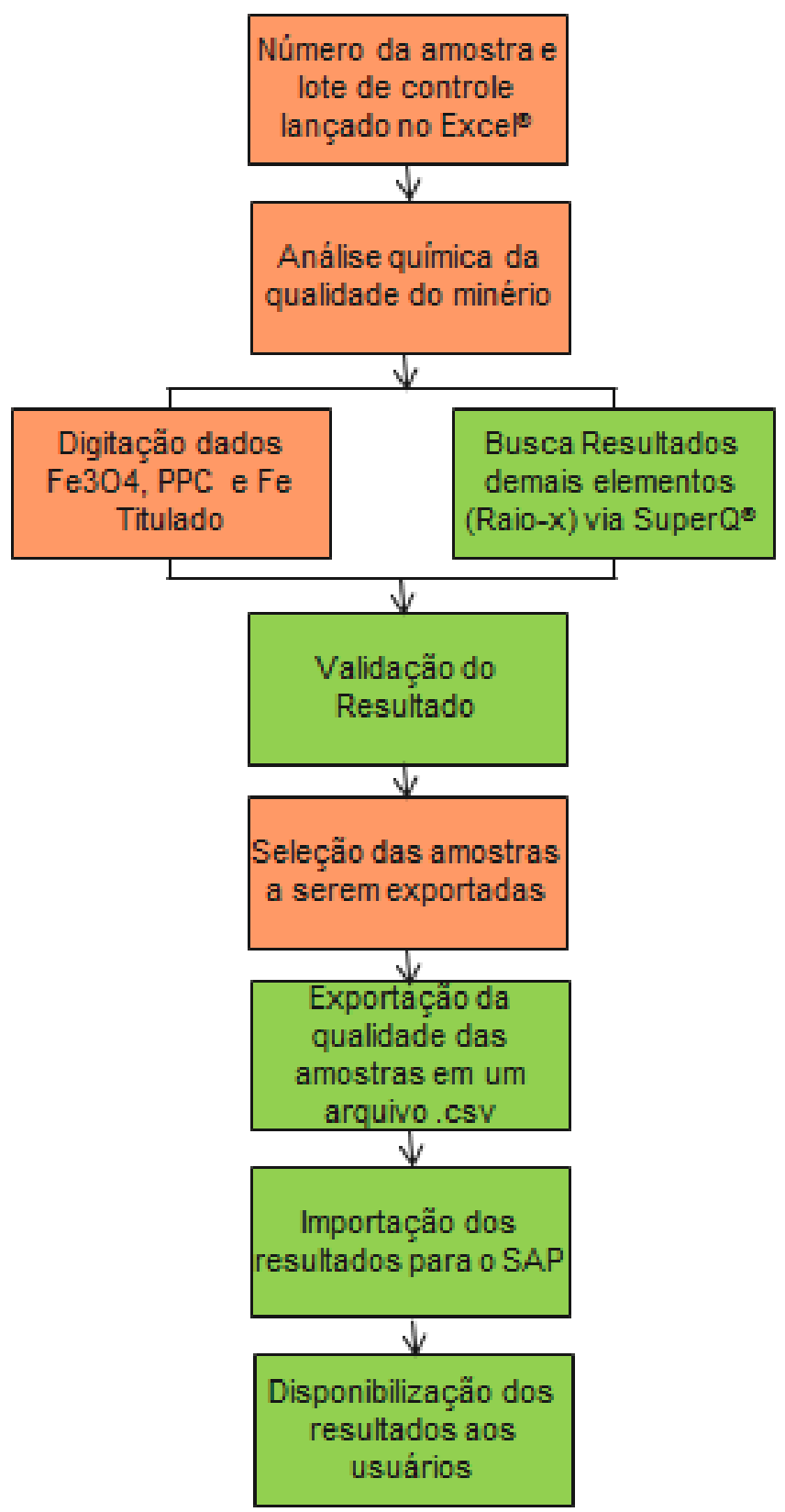

Figura 3 - Esquema de envio automático de dados 


\subsection{Verificação das análises}

O desenvolvimento dessa ferramenta viabilizou a inserção de algumas etapas de verificação automática para cada amostra, aumentando assim a agilidade e confiabilidade dos resultados.

\subsubsection{Estequiometria}

O fechamento estequiométrico de cada amostra é calculado utilizando-se dos diferentes teores analisados e avaliado de acordo com uma faixa pré-determinada para cada tipo de material; Caso a amostra analisada não esteja dentro da respectiva faixa, o analista é informado e a amostra deve ser reanalisada.

\subsubsection{Coerência}

A coerência dos resultados obtidos nas amostras de produto final é avaliada através de comparação com um histórico calculado mensalmente e embasado nos últimos seis meses de produção. Deste histórico são excluídos outliers e ajustados limites através de modelos matemáticos para 95\% das observações.

\begin{tabular}{|c|c|c|c|c|c|c|c|c|c|c|c|}
\hline \multirow{3}{*}{ OGF } & \multirow{2}{*}{ Elemento } & \multicolumn{2}{|c|}{$\mathrm{Fe}$} & \multicolumn{2}{|c|}{$\mathrm{SiO} 2$} & \multicolumn{2}{|c|}{$\mathbf{P}$} & \multicolumn{2}{|c|}{ Al203 } & \multicolumn{2}{|c|}{ PPC } \\
\hline & & LS & LI & LS & LI & LS & LI & LS & LI & LS & LI \\
\hline & Limites & 58,9644 & 55,8074 & 9,78633 & 3,73647 & 0,12514 & 0,05158 & 3,6728 & 1,16934 & 9,33325 & 6,54132 \\
\hline
\end{tabular}

Figura 4 - Exemplo fictício de limites de coerência de resultados utilizados

Caso a amostra seja reprovada nessa avaliação, ela segue um fluxo de aprovação. Primeiramente é realizado um contato telefônico com a equipe do beneficiamento sobre o resultado encontrado e verifica-se se esse valor é justificável ou esperado. Em caso positivo o resultado é aceito e a justificativa é anotada em um campo específico na planilha. Em caso negativo, a amostra parte para um fluxo de reanálise, detalhado a seguir.

\subsubsection{Reanálise}

Caso a amostra não esteja coerente com histórico e não houver justificativas operacionais que expliquem a divergência observada no resultado, a amostra deve ser reanalisada para validação do resultado. Para tal, a ferramenta utiliza-se do seguinte método:

Análise e reanálise são comparadas, teor por teor, e a diferença entre as duas análises são comparadas aos limites definidos anteriormente por estudo de ReR (Repetitividade e Reprodutibilidade) do laboratório. Em caso de aprovação, a média aritmética dos resultados é liberada para lançamento. Caso a diferença entre as duas leituras supere a referência estabelecida pelo estudo de ReR do laboratório em qualquer um dos teores analisados, é solicitada uma terceira análise. 
Após realização da terceira análise, seu resultado é comparado com o primeiro e segundo resultados obtidos anteriormente. Se houver coerência entre qualquer um dos pares (diferença entre as leituras menor a referência estabelecida pelo $\mathrm{ReR}$ ), a média destes é liberada, se não, uma quarta análise deve ser feita.

Assim como antes, o quarto resultado é comparado com os demais e se houver coerência entre algum deles, a média é liberada para lançamento. Caso não ocorra nenhuma coerência dentro dos limites estabelecidos, é feita a média entre os resultados centrais (mediana) e esse valor é liberado para ser enviado ao $\mathrm{SAP}^{\circledR}$.

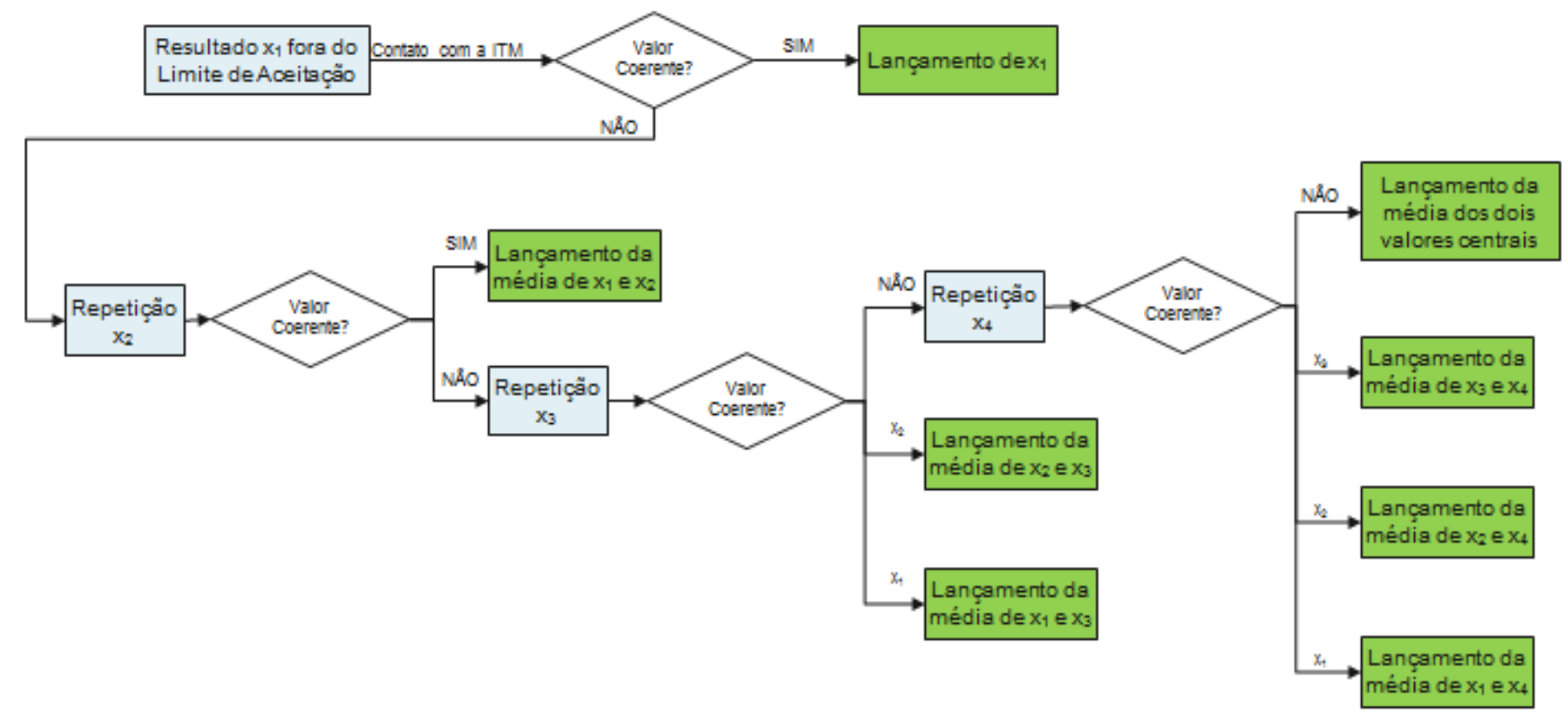

Figura 5 - Esquema de reanálise

Após a realização da reanálise, gera-se um arquivo PDF (Portable Document Format) com a identificação da amostra, resultados obtidos nas repetições, resultado de lançamento automático no SAP, fechamento estequiométrico e identificação do analista.

USIMINAS U

Avaliação de resultados de reanálise

$29 / 04 / 2017$ 10:32

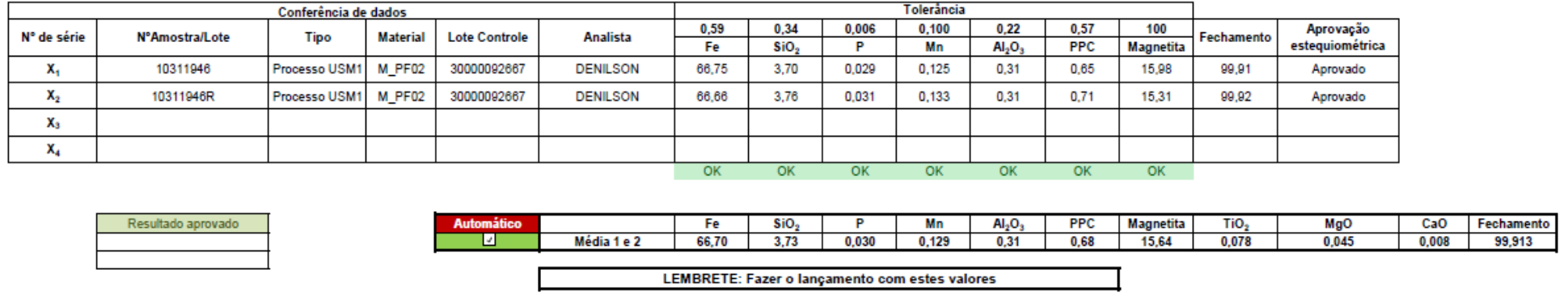

Figura 6 - Exemplo do arquivo em formato PDF de uma reanálise 


\subsection{Gestão das Análises}

A única parte da planilha Exce ${ }^{\circledR}$ cuja entrada de dados permanece manual consiste na entrada de dados para a identificação das amostras no inicio do processo, como o nome do analista, e informações necessárias à identificação da amostra, o que possibilita a geração de um boletim diário impresso para fins de auditoria, com todos os resultados de análises realizadas.

\section{USIMINAS Boletim de Análise Química}

\begin{tabular}{c|c|c|c|c|c|c|}
\hline $\begin{array}{c}N^{0} \text { de } \\
\text { Série }\end{array}$ & No Amostra Fisica $^{\circ}$ & Material & Tipo & $\begin{array}{c}\text { Centro / NF } \\
\text { (embarque) }\end{array}$ & Lote Controle & Analista \\
\hline 1 & & & & & & \\
\hline 2 & & & & & & \\
\hline 3 & & & & & & \\
\hline 4 & & & & & & \\
\hline \multicolumn{7}{r}{ Figura 7 - Campos de identificação manual da amostra } \\
\hline
\end{tabular}

\subsection{Análise de ferro}

Os diversos materiais analisados no laboratório possuem demandas diferentes de análise de ferro, em função dos diferentes níveis de precisão exigidos nos resultados. Alguns dos materiais, como os provenientes de processos do beneficiamento, podem ter o seu ferro calculado através da diferença no fechamento estequiométrico da amostra. Outros, como os materiais advindos de frente de lavra, podem ter a análise normalizada. Desta forma no laboratório podem ser obtidos resultados de teor de ferro para as amostras utilizando mais de uma metodologia: cálculo, normalização, análise via fluorescência de raios-x ou o titulação.

Para abranger estas distintas possibilidades de determinação de teor de ferro na amostra, incluiu-se na planilha criada um campo onde o analista pode selecionar o tipo de análise de ferro efetuado. Este campo é variável em função do o tipo de amostra e somente pode assumir valores conforme a necessidade de cada material, conforme visualizado na figura 8.

\begin{tabular}{|c|c|}
\hline Material & $\begin{array}{c}\text { Análise de } \\
\text { Ferro }\end{array}$ \\
\hline Produção & \\
\hline & 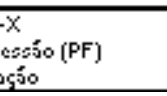 \\
\hline
\end{tabular}

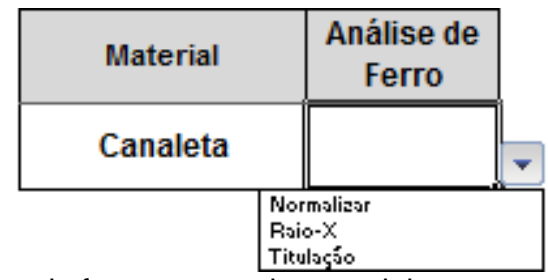

Figura 8 - Diferentes tipos de analise de ferro para cada material

\subsection{Outras melhorias}

Somente alguns materiais analisados na Mineração Usiminas possuem a necessidade de análise de Magnetita $\left(\mathrm{Fe}_{3} \mathrm{O}_{4}\right)$, de forma que antes 
da implantação desta ferramenta ocasionalmente os analistas se esqueciam de efetuar a análise. Por este motivo, foi incluído um esquema de verificação em relação a esse lançamento, alertando o analista através do aparecimento de um pop-up caso esse elemento esteja sem análise nas amostras que o necessitam.

Incluiu-se também um contador de amostra por turno, o que possibilita o acompanhamento da produtividade do laboratório, sendo possível saber quantas amostras de cada tipo foram analisadas por turno.

\section{RESULTADOS E DISCUSSÃO}

O lançamento automático das amostras de processo, que correspondem a aproximadamente $66 \%$ do total de amostras analisadas, promoveu redução do tempo gasto com digitação de resultados no sistema $\mathrm{SAP}^{\circledR}$, disponibilizando tempo do analista para execução de outras atividades no laboratório e reduzindo o risco de lançamentos incorretos em função de erro humano na digitação. Atualmente é realizado o lançamento de 2.118 amostras de forma automática, para um total de 3.221 amostras analisadas mensalmente. Isto representa uma economia de aproximadamente 106 horas mensais com a redução da digitação de resultados.

Com a criação de um esquema de verificação da qualidade da amostra baseado em um histórico de produção, obteve-se maior confiança e agilidade na interpretação dos resultados gerados no laboratório, visto que a avaliação da coerência do resultado tornou-se menos dependente da experiência e subjetividade do analista, evitando-se assim retrabalhos desnecessários e facilitando a identificação de incoerências.

\begin{tabular}{|c|c|c|c|c|c|c|c|c|}
\hline \multirow{2}{*}{\multicolumn{5}{|c|}{$\begin{array}{c}\text { Amostras de Prodeçáo-Processo } \\
\text { Conferêneia de dados }\end{array}$}} & \multicolumn{4}{|c|}{ Análise Quimioa [\%] } \\
\hline & & & & & $\mathrm{Fe}$ & $\mathrm{Fe}_{3} \mathrm{O}_{4}$ & $\mathrm{SiO}_{2}$ & P \\
\hline$\frac{N^{*} \text { serie }}{13}$ & $\begin{array}{l}\text { N-Amostia } \\
81164874\end{array}$ & Material & $\begin{array}{c}\text { Tiрo } \\
F+1,0\end{array}$ & Aotist de Terro & 6488 & 4.62 & 4.79 & 0005 \\
\hline 14 & 81164875 & Canaleta & $F+0.15$ & Normalizat & 57.72 & 4,50 & 5.75 & 0.053 \\
\hline 15 & 81164876 & Canaleta & $F-0.15$ & Normalizat & 36.38 & 0,99 & 44.86 & 0.052 \\
\hline 16 & 10316950 & Processo & MLSFTN & Titulaç5o & 60.54 & 9.77 & 7.11 & 0.070 \\
\hline 17 & 10316952 & Processo & M_PF02 & Regress 5o [PF] & 66,16 & 9.46 & 3.74 & 0.035 \\
\hline 18 & DGG3005173 & Produçăo & OGG & Titulap̧5o & 56.83 & & 6.04 & 0.145 \\
\hline 19 & OGF3005173 & Produç5o & OGF & Titulac5o & 57,61 & & 5,58 & 0.116 \\
\hline 20 & 10316953 & Processo & M_ALPO & Calculado & 51.66 & 5,45 & 17.98 & 0.081 \\
\hline 21 & 10316954 & Processo & M_ALRO & Calculado & 59,49 & & 7,56 & 0,091 \\
\hline 22 & 10316955 & Processo & M $\_$LSC & Calculado & 47,83 & 0,76 & 26,95 & 0,074 \\
\hline 23 & 10316956 & Processo & M_ALJG & Calculado & 59,69 & & 4,84 & 0,097 \\
\hline 24 & 10316957 & Processo & M_ALMA & Calculado & 60,03 & 8,84 & 7,25 & 0,079 \\
\hline 25 & 10316958 & Processo & M ALMF & Caloulado & 56,25 & 5,34 & 12,35 & 0,077 \\
\hline 26 & 10316966 & Processo & M_COMA & Caloulado & 65,58 & 16,65 & 3,35 & 0,048 \\
\hline 27 & 10316968 & Processo & M_COMF & Calculado & 66,13 & 11,60 & 3,39 & 0,038 \\
\hline 28 & 10316972 & Processo & M_COES & Calculado & 66,12 & & 2,36 & 0,043 \\
\hline 29 & 10316970 & Processo & M_COJG & Calculado & 56,74 & & 8,91 & 0,105 \\
\hline 30 & 10316959 & Processo & M_RJSC & Calculado & 51.84 & & 15,11 & 0,099 \\
\hline 31 & 10316960 & Processo & M_RJWH & Calculado & 33,18 & 1,08 & 45,22 & 0,075 \\
\hline 32 & 10316829 & Processo & M_UFES & Calculado & 49,08 & 1,60 & 76,61 & $0, m$ \\
\hline 33 & 10316674 & Processo & M_SFTN & Paio-X & 62,59 & 9,97 & 5,32 & 0,066 \\
\hline
\end{tabular}

\begin{tabular}{|c|c|c|c|c|}
\hline $\begin{array}{c}\text { Fecham } \\
\text { ento }\end{array}$ & $\begin{array}{c}\text { Fech. } \\
\text { Normali. }\end{array}$ & $\begin{array}{c}\text { Apromsio } \\
\text { estequiesétrice }\end{array}$ & $\begin{array}{c}\text { Aprovaça Jo } \\
\text { histórico }\end{array}$ & Autom \\
\hline 00.51 & & Aprovado & N5o se agloa & 口 \\
\hline 99.80 & 99.80 & Aprovado & NSo se aplica & $\square$ \\
\hline 100.20 & 100.20 & Aprovado & NSo se aplica & 口 \\
\hline 99.83 & & Aprovado & Reprovado & (7) \\
\hline 99.99 & & Aprovado & Aptowado & ( \\
\hline 100.21 & & Aprovado & Reprovado & 口 \\
\hline 100,36 & & Aprovado & Reprovado & D \\
\hline 100,00 & & Aprovado & N5o se aplioa & ( \\
\hline 100,00 & & Aprovado & N5o se aplioa & (7) \\
\hline 100,00 & & Aprovado & N5̃o se aplioa & (7) \\
\hline 100,00 & & Aprovado & N5o se aplioa & (7) \\
\hline 100,00 & & Aprovado & N5o se aplioa & (7) \\
\hline 100,00 & & Aprovado & N5o se aplioa & (7) \\
\hline 100,00 & & Aprovado & N5o se aplioa & (7) \\
\hline 100,00 & & Aprovado & N5o se aplioa & (7) \\
\hline 100,00 & & Aprovado & N5o se aplioa & (7) \\
\hline 100,00 & & Aprovado & N5̃o se aplioa & (7) \\
\hline 100,00 & & Aprovado & N5́o se aplioa & (7) \\
\hline 100,00 & & Aprovado & N5o se aplioa & (7) \\
\hline 100,00 & & Aprovado & N5o se aplioa & (7) \\
\hline 100,07 & & Aprovado & Aprowado & (7) \\
\hline
\end{tabular}

Figura 9 - Boletim de acompanhamento de Análise Química (dados fictícios) 
Com o desenvolvimento e implantação desta ferramenta tornou-se possível a gestão das análises em um só local, o que tornou mais simples o rastreamento das análises realizadas, facilitou a medição de produtividade por laboratorista e permitiu a geração de um registro impresso dos resultados para fins de auditoria de forma mais simples e em um formato padronizado.

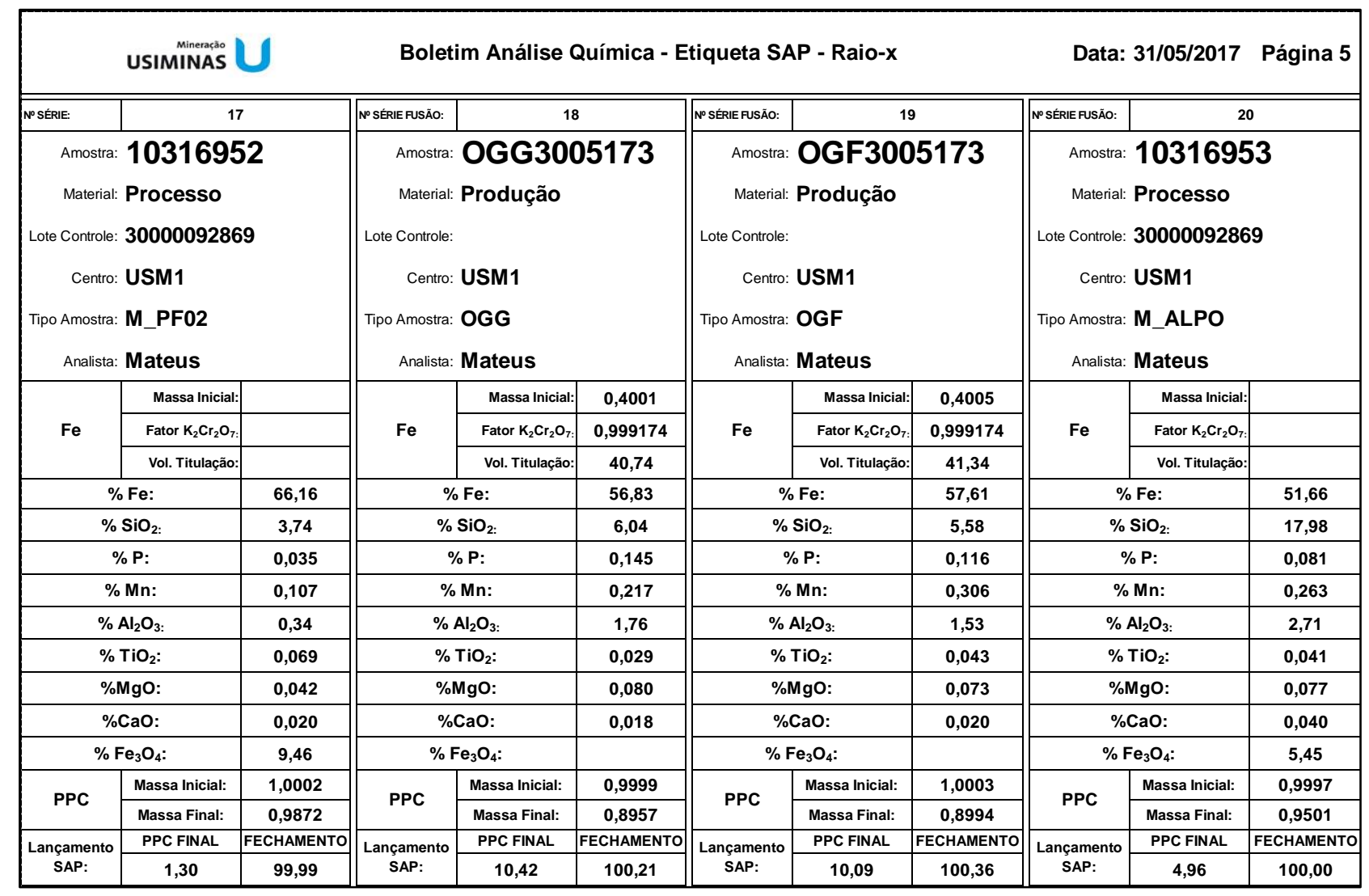

Figura 10 - Boletim para impressão dos resultados (dados fictícios)

\section{CONCLUSÃO}

A ferramenta apresentada neste trabalho atendeu às necessidades de rastreamento, verificação de resultados e redução do tempo despendido em lançamentos que motivaram 0 seu desenvolvimento, obtendo uma significativa melhora na gestão dos resultados e na robustez do controle das análises realizadas pelo laboratório da MUSA.

Conclui-se que os benefícios de controle das análises e aumento de confiabilidade advindos do desenvolvimento desta ferramenta podem ser obtidos em outras mineradoras de pequeno e médio porte, mesmo sem grandes investimentos em aquisição de software e serviços de terceiros, através do desenvolvimento de trabalhos similares.

\section{Agradecimentos}

Os autores agradecem à Mineração Usiminas pelo apoio no desenvolvimento e implantação desta ferramenta e na execução deste trabalho. 


\section{REFERÊNCIAS}

1 DIAS, Silvia M. P., PINTO, Elcio. A Utilização de um Sistema de Informação na Tomada de Decisões em uma Empresa Mineradora em Minas Gerais. ENEGEP. 2011. [acesso em 29 de maio de 2017]. Disponível em

http://www.abepro.org.br/biblioteca/enegep2011 TN STO 142898 18792.pdf

2 JANNUZZI, Celeste A. S. C., FALSARELLA, Orandi M., SUGAHARA, Cibele R.

Sistema de informação: um entendimento conceitual para a sua aplicação nas organizações empresariais. Perspect. ciênc. inf. vol.19 no 4 Belo Horizonte Oct./Dec. 2014. [acesso em 24 de maio de 2017]. Disponível em http://www.scielo.br/scielo.php?script=sci arttext\&pid=S1413-99362014000400007 SANTOS, Samantha E. V.; SANTOS, Joéfisson S. Contribuições de um Sistema de Gerenciamento de Informações e Automação de Laboratório: Estudo de Caso em uma Empresa de Mineração. ENEGEP. 2009. [acesso em 23 de maio 2017]. Disponível em http://www.abepro.org.br/biblioteca/enegep2009 TN STO 091615 13621.pdf SIQUEIRA, Marcelo C. Gestão Estratégica da Informação: como transformar o conteúdo informacional em conhecimento valioso. Rio de Janeiro: Brasport ; 2005. 